\title{
Influencia de las perforaciones corticales en regeneración ósea guiada: Revisión de la literatura
}

\section{The influence of cortical perforations on guided bone regeneration: A literature review}

\author{
Violeta Malpartida-Carrillo1*, Pedro Luis Tinedo-López¹, Maria Eugenia Guerrero ${ }^{1}$, Fernando Ortiz-Culca1
}

1. Maestría en Implantología Oral, Universidad Científica del Sur, Lima, Perú

* Correspondencia autor. Violeta Malpartida-Carrillo | Universidad Científica del Sur|Av. Paseo de la República $N^{\circ} 5544$ - Miraflores 18, Lima-Perú | Teléfono: (511) 6106400 Anx. 324 | E-mail: viletayu_30@hotmail.com

Trabajo recibido el 30/04/2018.

Aprobado para su publicación el 18/06/2018

\begin{abstract}
RESUMEN
Objetivo: Evaluar la influencia de las perforaciones corticales en regeneración ósea guiada (ROG) mediante resultados de histomorfometría obtenidos de estudios humanos y animales. Revisión de la literatura actual: Se realizó una búsqueda electrónica de estudios en humanos y animales en bases de datos PubMed, Cochrane Library, SciELO y Google Scholar desde 1980 hasta mayo del 2017. Se utilizó la siguiente estrategia de búsqueda: ((decortication of bone) OR (bone decortication) OR (alveolar decortication) OR (decortication) OR (decortications) OR (cortical perforation of bone) OR (cortical bone perforation) OR (cortical perforation) OR (cortical perforations) OR (intramarrow penetration) OR (marrow penetration)) AND ((guided bone regeneration) OR (guided bone augmentation) OR (bone augmentation) OR (osseous repair) OR (graft integration)). Discusión y conclusión: Se identificaron 65 artículos y sólo se seleccionaron 8. Cuatro estudios en animales mostraron influencia estadísticamente significativa al realizar perforaciones corticales. En 3 estudios en animales y en un estudio en humanos esta diferencia no fue estadísticamente significativa. Sin embargo, al realizar perforaciones existió mayor número de vasos sanguíneos y mayor formación ósea sin reacciones inflamatorias resaltantes ni impactos negativos.

Las perforaciones corticales muestran beneficios en etapas tempranas de cicatrización influyendo significativamente en la angiogénesis incrementando la cantidad de hueso neoformado.
\end{abstract}

PALABRAS CLAVE:

Regeneración ósea; Cicatrización de heridas; Histología.

Rev. Clin. Periodoncia Implantol. Rehabil. Oral Vol. 12(1); 37-40, 2019.

\section{ABSTRACT}

Objective: To evaluate the influence of cortical perforations on guided bone regeneration (ROG) by histomorphometry results obtained from human and animal studies. Review of current literature: An electronic search of trials in humans and animals with histomorphometric evaluations was carried out in the PubMed, Cochrane Library, SciELO and Google Scholar databases from 1980 to May 2017. The following search strategy was used: ((decortication of bone) OR (bone decortication) OR (alveolar decortication) OR (decortication) OR (decortications) OR (cortical perforation of bone) OR (cortical bone perforation) OR (cortical perforation) OR (cortical perforations) OR (intramarrow penetration) OR (marrow penetration)) AND ((guided bone regeneration) OR (guided bone augmentation) OR (bone augmentation) OR (osseous repair) OR (graft integration)). Discussion and conclusion: Sixty five articles were identified and only 8 were selected. Four studies in animals showed statistically significant influence when performing cortical perforations. In 3 studies in animals and in 1 human study this difference was not statistically significant. However, when drilling, a greater number of blood vessels and a greater bone formation without inflammatory reactions or negative impacts were recorded. Cortical perforations show benefits in early stages of healing, influencing significantly in the angiogenesis, increasing the amount of neoformed bone. KEY WORDS:

Bone regeneration; Wound healing; Histology.

Rev. Clin. Periodoncia Implantol. Rehabil. Oral Vol. 12(1); 37-40, 2019. 


\section{INTRODUCCIÓN}

Hoy en día, los implantes dentales representan una opción de tratamiento predecible en la rehabilitación oral de pacientes desdentados parciales y totales ${ }^{(1,2)}$. La mayor confiabilidad de su utilización se debe al avance en el desarrollo de superficies, diseños de implantes, técnicas quirúrgicas, técnicas protésicas y biomateriales. Sin embargo, a pesar de los distintos avances tecnológicos, la disponibilidad ósea es aún el prerrequisito principal para la colocación y rehabilitación exitosa de los implantes dentales ${ }^{(3)}$. El procedimiento de regeneración ósea guiada (ROG) ha sido utilizado por muchos años como una técnica para devolver el ancho y la altura ósea perdida propiciando la colocación de implantes dentales durante la realización de la técnica o en una segunda etapa quirúrgica ${ }^{(4,5)}$. Las perforaciones corticales, penetraciones intramedulares o decorticaciones se utilizan de manera común como parte del procedimiento de ROG y su fundamento biológico se basa en que permitirían una vía de fácil acceso para la llegada de células osteoprogenitoras, mesenquimales y vasos sanguíneos al sitio tratado facilitando rápida angiogénesis, y además mejorarían la unión o enlace físico entre el injerto y el lecho receptor $^{(6)}$. Algunos estudios han demostrado la efectividad de realizar perforaciones corticales durante la ROG mediante evaluaciones histológicas ${ }^{(7,8)}$; sin embargo, otros estudios no muestran beneficios de su utilización ${ }^{(9,10)}$. En la literatura dental, existe un debate en curso sobre la verdadera influencia de las perforaciones corticales en la ROG porque su capacidad para acelerar o aumentar el procedimiento muestra aún resultados contradictorios. Es importante resaltar que el análisis con histomorfometría es considerado el método gold estándar para estimar la cantidad de hueso neoformado, de tejido blando y de partículas del injerto residual en los sitios tratados con injertos ${ }^{(11)}$. En el año 2009, Greenstein et al. ${ }^{(12)}$ reportaron la influencia de las perforaciones corticales en la ROG con evaluaciones de histomorfometría en algunos estudios animales. Sin embargo, es importante conocer dicha influencia en estudios humanos asi como una actualización de los resultados en estudios animales.

Siendo así, el propósito de la presente revisión fue evaluar la influencia de las perforaciones corticales en la ROG mediante resultados de histomorfometría obtenidos de estudios humanos y animales.

\section{REVISIÓN DE LA LITERATURA ACTUAL}

Se realizó una búsqueda electrónica médico-dental en las bases de datos PubMed, Cochrane Library, SciELO y Google Scholar desde 1980 hasta mayo del 2017. Se utilizó la siguiente estrategia de búsqueda: ((decortication of bone) OR (bone decortication) OR (alveolar decortication) OR (decortication) OR (decortications) OR (cortical perforation of bone) OR (cortical bone perforation) OR (cortical perforation) OR (cortical perforations) OR (intramarrow penetration) OR (marrow penetration)) AND ((guided bone regeneration) OR (guided bone augmentation) OR (bone augmentation) OR (osseous repair) OR (graft integration)). La búsqueda electrónica se complementó con una búsqueda manual tomando en consideración la lista de referencias de las publicaciones incluidas.

\section{Selección de estudios: criterios de elegibilidad}

Se verificó la relevancia de los títulos y resúmenes de los artículos obtenidos para luego descargar el texto completo de los artículos pertinentes cuando era posible; de lo contrario, sólo se obtuvieron los resúmenes electrónicos.

\section{Criterios de inclusión:}

- Ensayos clínicos aleatorizados en humanos.

- Ensayos aleatorizados en animales.

- Evaluaciones con histomorfometría.

- Existencia de un grupo prueba y de un grupo control.

- Estudios sin restricción del idioma.

\section{Criterios de exclusión}

- Estudios con descripciones ambiguas del procedimiento.

- Estudios en elementos finitos, pilotos, reportes de caso y artículos de discusión.

$$
\text { - Estudios con resultados de un solo grupo evaluado. }
$$

\section{Recolección de datos:}

Se realizó una búsqueda exhaustiva de la literatura científica para identificar los estudios realizados en humanos y animales que evaluaron la influencia de las perforaciones corticales en la ROG utilizando histomorfometría.

Los datos fueron recolectados y analizados teniendo en consideración lo siguiente:

- Autor y año de publicación.

- Diseño de estudio.

- Tamaño de la muestra.
- Período de evaluación.

- Porcentaje del incremento óseo con y sin perforaciones corticales.

- Descripción del procedimiento.

La búsqueda electrónica inicial en las 4 bases de datos y la búsqueda manual arrojaron un total de 65 artículos. Luego de seleccionar los títulos se registraron 42 resúmenes. Posteriormente, al eliminar artículos duplicados y no relacionados se obtuvieron 30 artículos para la evaluación del texto completo. Basado en los criterios de elegibilidad se excluyeron 22 artículos. Finalmente, se obtuvieron 8 artículos para el análisis final (Figura 1). Los datos cuantitativos y cualitativos se extrajeron de los 8 artículos con texto completo (Tabla 1). De estos estudios, solo 1 evaluó las perforaciones corticales en humanos ${ }^{(13)}$, los otros 7 estudios $^{(6,14-19)}$ fueron realizados en defectos craneales de animales (conejos y ratas).

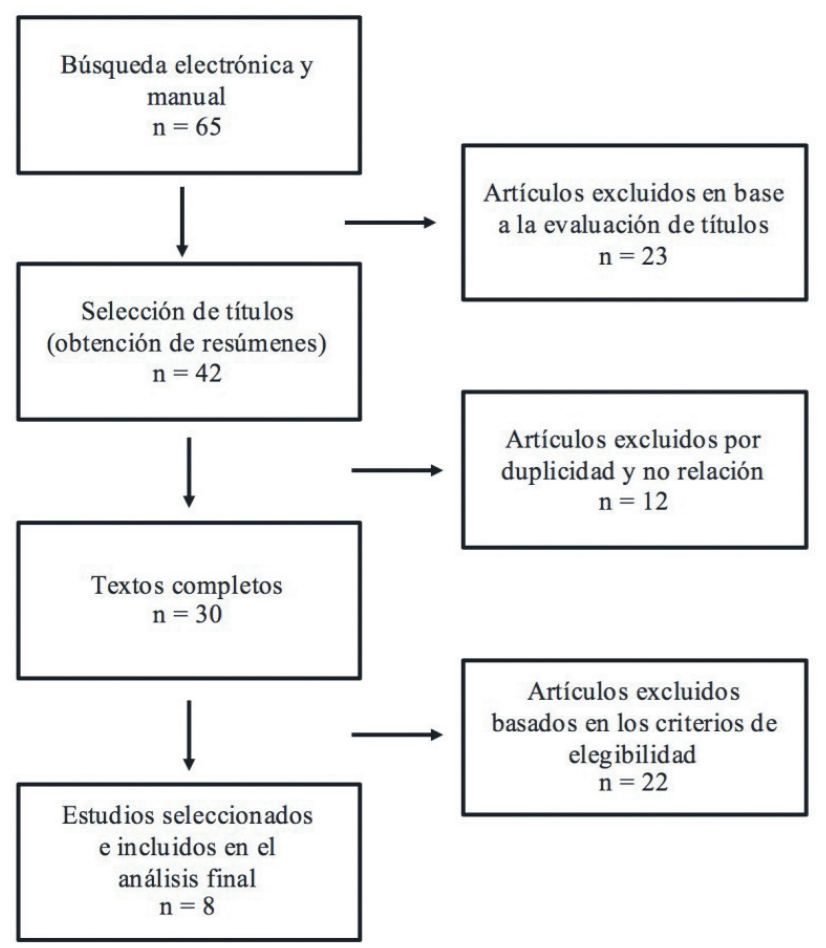

Figura 1. Estrategia de búsqueda utilizada para identificar los estudios incluidos.

\section{DISCUSIÓN Y CONCLUSIÓN}

El objetivo del presente artículo de revisión fue conocer la influencia de las perforaciones corticales en la ROG comparando los resultados de histomorfometría en los porcentajes de neoformación ósea al realizar o no dicha técnica en estudios humanos y animales.

Entre los artículos obtenidos para evaluación, se encontraron distintos términos para referirse al retiro intencional de partes de la cortical ósea durante la ROG. Estos términos fueron: perforaciones corticales $(6,13,15,17)$, penetraciones intramedulares ${ }^{(14)}$, penetraciones medulares ${ }^{(18)}$ y decorticaciones ${ }^{(16,19)}$. Los datos que confirman que las perforaciones corticales mejoraron de manera significativa los resultados de la ROG se han reportado en 4 estudios en animales ${ }^{(14,15,18,19)}$ y se muestran en la Tabla 1. Majzoub et al. ${ }^{(14)}$ diseñaron un estudio en 16 conejos donde luego de realizar perforaciones corticales o no sobre defectos craneales, colocaron cúpulas de titanio como barreras oclusivas. Luego de 60 días, la evaluación histomorfométrica demostró mayor porcentaje de neoformación ósea en el grupo que recibió perforaciones $(71.7 \%$ versus $53.5 \%)$. En un diseño de estudio semejante, Min et al..$^{(18)}$ evaluaron los efectos de las perforaciones medulares en 10 conejos y luego de 3 meses los autores encontraron mayor porcentaje de neoformación ósea en el grupo con perforaciones (78.9\% versus $69.8 \%)$. Rompen et al. ${ }^{(15)}$ evaluaron la influencia de las perforaciones corticales en 30 ratas utilizando cámaras de titanio como barreras oclusivas. Luego de 16 semanas encontraron mayor porcentaje de hueso nuevo en el grupo con perforaciones (172.8\% versus $141 \%)$. En el año 2016. Acar et al. ${ }^{(19)}$ ejecutaron un estudio en 16 conejos para conocer si tiene algún efecto el mayor número de perforaciones en la 
Tabla 1. Evaluación de los artículos seleccionados e incluidos en el análisis final.

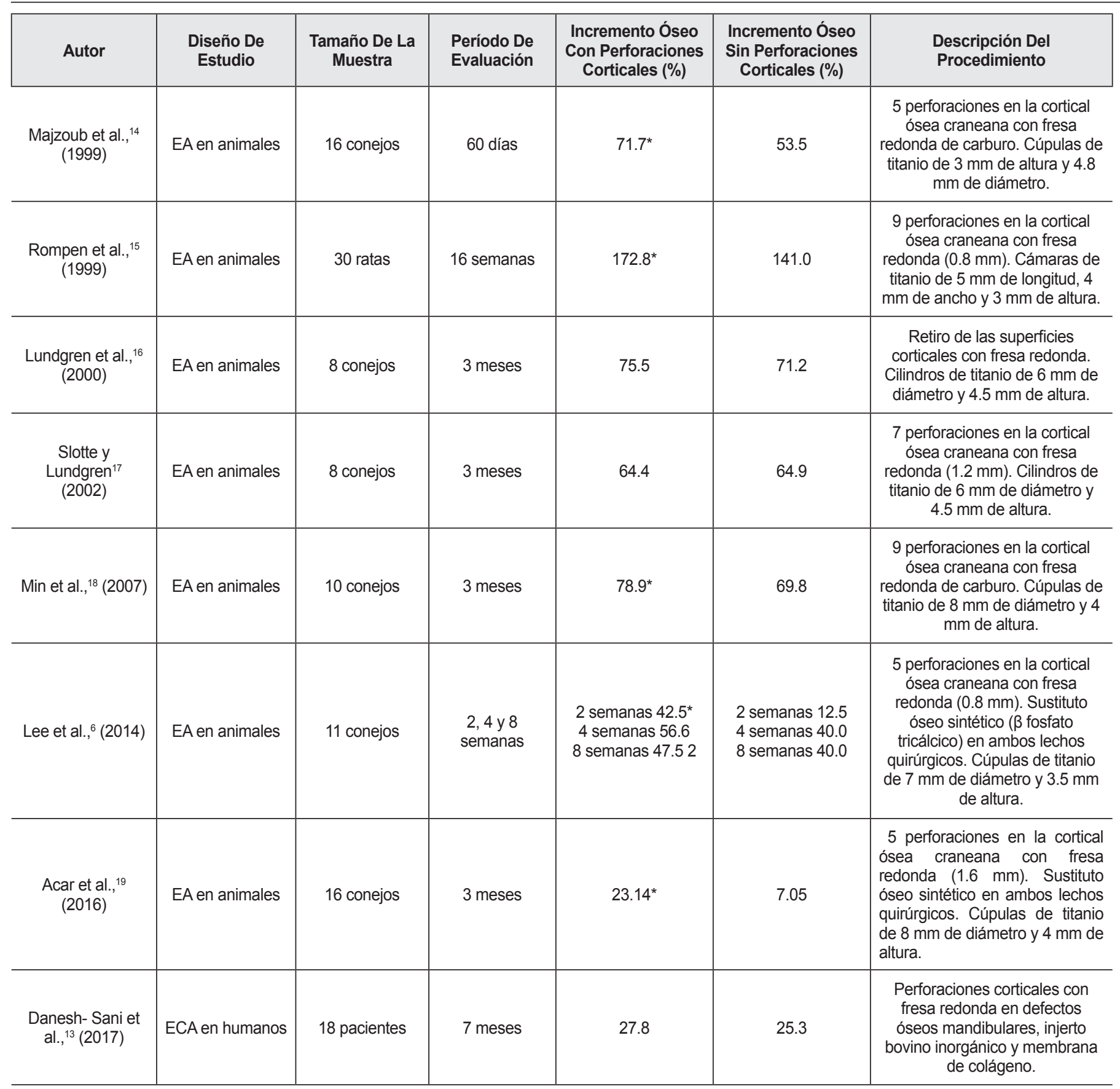

EA: Ensayo Aleatorizado, ECA: Ensayo Clínico Aleatorizado. *Diferencia estadísticamente significativa.

cortical. Según el diseño de estudio, los autores colocaron hueso sintético sobre los sitios perforados o no y los sellaron utilizando cúpulas de titanio. A finales del tercer mes observaron mayor porcentaje de neoformación ósea en el grupo que recibió perforaciones (23.1\% versus $7.1 \%)$. Sin embargo, no observaron mayor efecto significativo al aumentar el número de estas preparaciones.

Los datos que confirman que las perforaciones corticales no mejoraron de manera significativa los resultados de la ROG se han reportado en 3 estudios en animales ${ }^{(6,16,17)}$ y en un estudio en humanos ${ }^{(13)}$ y se muestran en la Tabla 1. Lundgren et al. ${ }^{(16)}$ evaluaron la influencia de la decorticación en la ROG realizada en 8 conejos utilizando cilindros de titanio como barreras oclusivas. Luego de 3 meses, los autores no encontraron diferencias significativas entre los porcentajes de neoformación ósea $(75.5 \%$ versus $71.2 \%$ ); sin embargo, se observó mayor formación en el grupo perforado. En un diseño de estudio semejante, Slotte y Lundgren ${ }^{(17)}$ también evaluaron el impacto de las perforaciones corticales en 8 conejos. Luego de 3 meses, no encontraron diferencias significativas entre una y otra técnica $(64.4 \%$ versus 64.9\%). En el año 2014, Lee et al.(6) evaluaron la influencia de las perforaciones corticales en la ROG utilizando $\beta$ fosfato tricálcico con cúpulas de titanio sobre los lechos perforados o no en 11 conejos. Luego de 8 semanas no encontraron diferencias significativas en el porcentaje de neoformación ósea entre los grupos ( $47.5 \%$ versus $40 \%$ ); sin embargo, existió mayor formación en el grupo perforado. Adicionalmente, dicha diferencia sí fue significativa en la segunda semana $(42.5 \%$ versus $12.5 \%$ ) por lo que los autores resaltaron el efecto de las perforaciones en la angiogénesis incrementando la cantidad de hueso neoformado en las fases iniciales de cicatrización ósea. Recientemente, en un ensayo clínico aleatorizado y controlado, Danesh-Sani et al. ${ }^{(13)}$ evaluaron la influencia de las perforaciones corticales en la ROG en 18 pacientes con defectos óseos en la mandíbula. Se distribuyeron de manera aleatoria 9 pacientes para recibir perforaciones corticales con una fresa redonda más injerto de bovino y membrana de colágeno, siendo éste el grupo prueba. En el grupo control sólo se colocó el injerto y la membrana. Luego de 7 meses no hubo diferencia significativa en el porcentaje de neoformación ósea entre ambos grupos (27.8\% versus $25.3 \%$ ); sin embargo, fue mayor en el grupo perforado. Adicionalmente, se reportó incremento significativo 
en el número de nuevos vasos sanguíneos (angiogénesis) por lo que los autores resaltaron la influencia de las perforaciones en las etapas iniciales de formación ósea.

En la literatura se hace referencia al fenómeno regional acelerado producido por un "noxious stimuli" (estímulo nocivo) que aceleraría el proceso de cicatrización normal( ${ }^{(20,21)}$. Las perforaciones corticales pueden considerarse como tal estímulo ya que se realizan de manera intencional para producir un lecho sangrante que propicie rápida angiogénesis acelerando la cicatrización, así como para permitir una mejor conexión entre el injerto y el lecho receptor ${ }^{(12)}$. Es por ello la importancia de conocer los verdaderos efectos de realizar perforaciones corticales basados en histomorfometría. Según los resultados, la mayoría de estudios han reportado la influencia de las perforaciones en animales, utilizando el cráneo de conejos y ratas como lechos receptores. Sin embargo, se debe considerar la gran cantidad de foraminas naturales presentes en dichas localizaciones los cuales podrían influenciar los resultados de neoformación y de vascularización. Otra consideración a tener en cuenta es que dichos animales pertenecen a especies filogenéticas de escalas menores caracterizadas por un alto potencial osteogénico en comparación a especies animales de escalas mayores (monos o perros) ${ }^{(12,13)}$, por lo que sería de gran importancia la evaluación de las perforaciones corticales en estos últimos. Es importante resaltar que sólo un ensayo clínico en humanos reporta evaluaciones de las perforaciones corticales en la ROG utilizando histomorfometría ${ }^{(13)}$, necesitándose estudios futuros con este diseño de estudio. De Avila et al. ${ }^{(8)}$ reportaron una evaluación histológica sin histomorfometría realizando o no perforaciones corticales en los injertos en bloque de 10 pacientes. Luego de 6 meses, los autores evidenciaron la presencia de tejido conectivo fibroso en la interface entre el injerto y el lecho receptor de los pacientes en los cuales no se realizaron perforaciones en comparación a la completa integración observada en los pacientes en los que sí se realizaron perforaciones.

Considerando los resultados histomorfométricos más que la significancia estadística de los estudios incluidos, se puede resaltar que en 6 de los 7 estudios evaluados en animales el mayor porcentaje de incremento óseo siempre estuvo relacionado a la presencia de perforaciones corticales ${ }^{(6,14-16,18,19)}$. Esto también se demuestra en el único estudio reportado en humanos ${ }^{(13)}$. Adicionalmente, ningún estudio reporta reacciones inflamatorias resaltantes ni impactos negativos al realizar perforaciones en la cortical ósea. En cuanto a las características de las perforaciones corticales, no existe un consenso sobre cómo realizar el procedimiento ni sobre el tamaño y la cantidad de éstas. Nishimura et al. ${ }^{(22)}$ en un estudio en conejos no encontraron diferencia estadísticamente significativa al realizar perforaciones corticales de mayor tamaño $(1 \times 15$ $\mathrm{mm}$ versus $3 \times 15 \mathrm{~mm}$ ). Así mismo, Acar et al. ${ }^{(19)}$ no evidenciaron diferencia estadísticamente significativa al realizar mayores perforaciones en la cortical (5 perforaciones versus 1 perforación). Sin embargo, los resultados histomorfométricos de ambos estudios muestran mayor formación ósea al realizar perforaciones de mayor tamaño $\left(12.75 \mathrm{~mm}^{2}\right.$ versus $\left.9.70 \mathrm{~mm}^{2}\right)$ (22) y al realizar mayor número de éstas $(23.1 \% \text { versus } 18.9 \%)^{(19)}$. Los resultados contradictorios observados en los estudios evaluados pueden estar relacionados a diferencias en el diseño de estudio, en el tiempo de evaluación y en las distintas especies evaluadas. Es interesante resaltar que en todos los estudios realizados en animales ${ }^{(6,14-19)}$ y en el único estudio realizado en humanos ${ }^{(13)}$ se utilizaron fresas redondas pequeñas entre $0.8 \mathrm{~mm}$ y $1.6 \mathrm{~mm}$ para realizar las perforaciones por lo que es de uso común para el procedimiento.

Es difícil evaluar en humanos la cicatrización ósea en sus fases iniciales ya que se requiere regenerar un lecho para que luego de su integración se pueda obtener una biopsia antes de la colocación de implantes. Los estudios en animales que evaluaron tiempos iniciales de cicatrización pueden promovernos información adicional sobre la influencia de las perforaciones en etapas iniciales de la ROG. Si los resultados son semejantes en etapas iniciales y finales, las perforaciones corticales podrían no mostrar su utilidad.

Según los resultados de evaluaciones histomorfométricas, las perforaciones corticales muestran beneficios en etapas tempranas de cicatrización ósea influyendo de manera significativa en la angiogénesis incrementando así la cantidad de hueso neoformado en el procedimiento de ROG.

\section{RELEVANCIA CLÍNICA}

Regeneración ósea guiada (ROG) es un tratamiento común realizado en Periodoncia e Implantología Oral. De manera clásica se recomienda realizar perforaciones corticales antes de ROG para mejorar la angiogénesis y la conexión física entre el injerto y el lecho receptor. Sin embargo, algunos autores mencionan que dichas perforaciones no muestran beneficios en el resultado final. Considerando que el análisis con histomorfometría es el método gold estándar para estimar la cantidad de hueso neoformado, sería de gran utilidad conocer la influencia de las perforaciones corticales en ROG a partir de resultados histomorfométricos para tener un aporte científico sobre su utilidad práctica.

\section{CONFLICTO DE INTERÉS}

Los autores declaran que no tienen ningún conflicto de interés.

\section{Bibliografía}

1. Seiler I, Mühlemann S, Zwahlen M, Hämmerle $\mathrm{CH}$, Schneider D. Cemented and screw-retained implant reconstructions: a systematic review of the survival and complication rates. Clin Oral Implants Res. 2012;23:163-201.

2. Wittneben JG, Millen C, Brägger U. Clinical performance of screw-versus cementretained fixed implant-supported reconstructions - a systematic review. Int J Oral Maxillofac Implants. 2014;29:84-98

3. Sanz-Sánchez I, Ortiz-Vigón A, Sanz-Martín I, Figuero E, Sanz M. Effectiveness of lateral bone augmentation on the alveolar crest dimension: a systematic review and meta-analysis. J Dent Res. 2015;9:128-42.

4. Urban IA, Nagursky H, Lozada JL, Nagy K. Horizontal ridge augmentation with a collagen membrane and a combination of particulated autogenous bone and anorganic bovine bone-derived mineral: a prospective case series in 25 patients. Int J Periodontics Restorative Dent. 2013;33:299-307.

5. Urban IA, Lozada JL, Jovanovic SA, Nagursky H, Nagy K. Vertical ridge augmentation with titanium-reinforced, dense-PTFE membranes and a combination of particulated autogenous bone and anorganic bovine bone-derived mineral: a prospective case series in 19 patients. Int J Oral Maxillofac Implants. 2014;29:185-93. 6. Lee SH, Lim P, Yoon HJ. The influence of cortical perforation on guided bone regeneration using synthetic bone substitutes: a study of rabbit cranial defects. Int J Oral Maxillofac Implants. 2014;29:464-71.

7. Cha JK, Kim CS, Choi SH, Cho KS, Chai JK, Jung UW. The influence of perforating the autogenous block bone and the recipient bed in dogs. Part II: histologic analysis. Clin Oral Implants Res. 2012;23:987-92.

8. de Avila ED, Filho JS, de Oliveira Ramalho LT, Real Gabrielli MF, Pereira Filho VA. Alveolar ridge augmentation with the perforated and nonperforated bone grafts J Periodontal Implant Sci. 2014;44:33-8.

9. Barbosa DZ, de Assis WF, Shirato FB, Moura CC, Silva CJ, Dechichi P. Autogenous bone graft with or without perforation of the receptor bed: histologic study in rabbit calvaria. Int J Oral Maxillofac Implants. 2009;24:463-8.

10. Dayangac E, Araz K, Oguz Y, Bacanli D, Caylak B, Uckan S. Radiological and histological evaluation of the effects of cortical perforations on bone healing in mandibular onlay graft procedures. Clin Implant Dent Relat Res. 2016;18:82-8.

11. Chackartchi T, lezzi G, Goldstein M, Klinger A, Soskolne A, Piattelli A, et al. Sinus floor augmentation using large $(1-2 \mathrm{~mm})$ or small $(0.25-1 \mathrm{~mm})$ bovine bone minera particles: a prospective, intra-individual controlled clinical, micro-computerized tomography and histomorphometric study. Clin Oral Implants Res. 2011:22:473-80.
12. Greenstein G, Greenstein B, Cavallaro J, Tarnow D. The role of bone decortication in enhancing the results of guided bone regeneration: a literature review. J Periodontol. 2009;80:175-89.

13. Danesh-Sani SA, Tarnow D, Yip JK, Mojaver R. The influence of cortical bone perforation on guided bone regeneration in humans. Int J Oral Maxillofac Surg. 2017; $46: 261-6$

14. Majzoub Z, Berengo M, Giardino R, Aldini NN, Cordioli G. Role of intramarrow penetration in osseous repair: a pilot study in the rabbit calvaria. J Periodontol. 1999;70:1501-10.

15. Rompen EH, Biewer R, Vanheusden A, Zahedi S, Nusgens B. The influence of cortical perforations and of space filling with peripheral blood on the kinetics of guided bone generation. A comparative histometric study in the rat. Clin Oral Implants Res. 1999;10:85-94.

16. Lundgren AK, Lundgren D, Hämmerle $\mathrm{CH}$, Nyman S, Sennerby L. Influence of decortication of the donor bone on guided bone augmentation. An experimental study in the rabbit skull bone. Clin Oral Implants Res. 2000:11:99-106.

17. Slotte C, Lundgren D. Impact of cortical perforations of contiguous donor bone in a guided bone augmentation procedure: an experimental study in the rabbit skull. Clin Implant Dent Relat Res. 2002;4:1-10.

18. Min S, Sato S, Murai M, Okuno K, Fujisaki Y, Yamada Y, et al. Effects of marrow penetration on bone augmentation within a titanium cap in rabbit calvarium. J Periodontol. 2007;78:1978-84.

19. Acar $A H$, Alan H, Özgür C, Vardi N, Asutay F, Güler C. Is more cortical bone decortication effective on guided bone augmentation?. J Craniofac Surg. $2016 ; 27^{(7)}: 1879-83$

20. Frost HM. The biology of fracture healing. An overview for clinicians. Part I. Clin Orthop Relat Res. 1989;248:283-93.

21. Frost HM. The biology of fracture healing. An overview for clinicians. Part II. Clin Orthop Relat Res. 1989:248:294-309.

22. Nishimura I Shimizu Y, Ooya K. Effects of cortical bone perforation on experimental guided bone regeneration. Clin Oral Implant Res. 2004;15(3):293-300 